Vol. 19(2):134-141, agosto - noviembre 2016

\title{
Formación por competencias en Enfermería. Experiencia de la Universidad de Chile
}

\author{
Training by Competencies in Nursing. An Experience from the \\ University of Chile
}

\section{Formação por competências na enfermagem. Experiência da Universidade do Chile}

Amalia Silva-Galleguillos, Enf., MSc., PhD. *

\begin{abstract}
Resumen
Introducción: La Escuela de Enfermería de la Universidad de Chile en el año 2006, inició el cambio de plan de formación de un currículo por objetivos a uno basado en competencias, implementación que se inició en el año 2013. Objetivo: Este artículo busca presentar una síntesis del trabajo realizado desde el año 2010 al 2013 por la comisión de innovación curricular de la Escuela de Enfermería Universidad de Chile para lograr implementar un currículo basado en competencias. Metodología: Se presentan las distintas fases que exigen la integración de diferentes actores, a saber; el cuerpo académico de la escuela, profesores externos a la unidad, empleadores, egresados y estudiantes de la carrera de enfermería. Conclusiones: Esta experiencia permitió reflexionar sobre algunos de los cambios de paradigmas requeridos en este proceso como son; el estudiante en el centro del aprendizaje, con participación dinámica y proactiva de los mismos, el establecimiento de un lenguaje diferente, con metodologías innovadoras que apunten a un aprendizaje significativo. [Silva-Galleguillos A. Formación por competencias en Enfermería. Experiencia de la Universidad de Chile.MedUNAB 2016; 19(2): 134-141].
\end{abstract}

Palabras clave: Educación basada en competencias; Enfermería; Aprendizaje; Estudiantes; Educación.

\begin{abstract}
Introduction: In 2006, the Nursing School of the University of Chile began changing the teaching plan from a learning objective syllabus to one based on competencies; this implementation began in 2013. Objective: This paper aims to present a summary of a research carried out from 2010 to 2013 by the syllabus innovation commission of the Nursing School from the University of Chile in order to implement a syllabus based on competencies Methodology: It presents the different phases that require the integration of different actors, per se; the academic school staff, external teachers to it, employers, graduates and students from the nursing program as well. Conclusions: This experience allowed thinking about some of the paradigm changes required in this process, such as the student at the learning center, with dynamic and proactive participation of them, the agreement on using a different language, with innovative methodologies that aim to a meaningful learning. [Silva-Galleguillos A. Training by Competencies in Nursing. An Experience from the University of Chile. MedUNAB 2016; 19(2): 134-141].
\end{abstract}

Keywords: Competency-based education; Nursing; Learning; Students; Education.

\footnotetext{
* Enfermera, Magister en salud Pública Internacional, Magister en Investigación en Cuidados de Enfermería, Experto Universitario en Salud y Cooperación al Desarrollo, Directora del Departamento de Enfermería 2014-2016 Profesor asistente Escuela de Enfermería, BPSO Chile, Encargada Comisión Innovación Curricular 2011-2013 Escuela de Enfermeria Universidad de Chile, Santiago de Chile, Chile.
}

Autor de Correspondencia: Amalia Silva Galleguillos, Departamento de Enfermería, Universidad de Chile, Facultad de Medicina Av. Independencia 1027 Santiago de Chile, Chile. Correo electrónico: asilva@med.uchile.cl 


\section{Resumo}

Introdução: A Escola de Enfermagem da Universidade do Chile, em 2006, deu início à mudança do currículo de formação desenhado por objetivos, para outro baseada em competências e sua aplicação começou em 2013. Objetivo: Este artigo procura apresentar uma síntese do trabalho realizado desde 2010 a 2013, mediante a prática de inovação curricular da Escola de Enfermagem da Universidade do Chile, ao implementar com sucesso um currículo baseado em competências. Temas: $\mathrm{O}$ artigo apresenta as diferentes fases que exigem a integração de diferentes atores, ou seja;

\section{Introducción}

Los cambios curriculares se han desarrollado en diferentes lugares del mundo, básicamente atendiendo a una serie de fenómenos como la globalización, los avances tecnológicos, nuevas necesidades y demandas de la sociedad (1), cambios en las características de estudiantes (2) y la democratización del conocimiento por medio del internet. La enfermería chilena también ha visto la necesidad de adaptarse a los cambios antes mencionados, replanteándose el perfil que deben poseer los nuevos profesionales acorde al contexto del país y de un mundo cada vez más globalizado, donde la migración de profesionales en busca de mejores condiciones de desempeño es un tema contingente.

La reforma curricular basada en competencias, se realizó desde el modelo utilizado en el proceso de Bolonia, el proceso por competencias que guía la reforma en Chile y Latinoamérica es el desprendido del Proceso Bolonia, en Europa (Tuning). Sin desconocer que el cambio curricular también ha ocurrido en países como Canadá, Australia y Nueva Zelanda (3). Este artículo tiene como objetivo reflexionar sobre la experiencia de la implementación de un currículo por competencias en la Escuela de Enfermería de la Universidad de Chile.

\section{Competencias y modelos de aprendizaje}

Las competencias son habilidades complejas, las cuales reflejan el saber hacer en una situación específica (4), no obstante, en su complejidad están involucrados aspectos éticos, de identidad profesional y personal, fortaleciendo habilidades de pensamiento complejo y reflexivo (5). El modelo de aprendizaje por competencias es un proceso educativo centrado en el estudiante. Su objetivo primordial es que el estudiante llegue a ser "competente" frente a las situaciones y contextos que deberá enfrentar durante su vida profesional, en otras palabras, el estudiante deberá ser capaz de recurrir a todos los recursos con los que cuenta permitiéndole integrar los conocimientos y habilidades en el desarrollo de su carrera y posterior a su egreso. El proceso por competencias que guía la reforma en Chile y corpo docente da escola, professores externos à unidade, os empregadores, ex-alunos e estudantes da carreira de enfermagem. Conclusões: Esta experiência permitiu refletir sobre algumas mudanças de paradigma, necessárias neste processo, isto é: o aluno no centro de aprendizagem com participação dinâmica e proativa, o uso da linguagem diferente, com metodologias inovadoras destinadas a aprendizagem significativa. [Silva-Galleguillos A. Formação por competências na enfermagem. Experiência da Universidade do Chile. MedUNAB 2016; 19(2): 134-141].

Palavras-chave: Educação baseada em competências; Enfermagem; Aprendizagem; Estudantes; Educação.

Latinoamérica es el desprendido del Proceso Bolonia en Europa (Tuning). Sin desconocer que el cambio curricular también ha ocurrido en países como Canadá, Australia y Nueva Zelanda (2).

El proyecto Tuning es una iniciativa que tiene como objetivo concreto implementar el proceso de Bolonia en las instituciones de educación superior. Consiste en una metodología de rediseño, desarrollo, implementación y evaluación de los programas de estudio de cada uno de los ciclos de Bolonia, esto puede ser considerado válido en todo el mundo ya que ha sido validado en distintos países y continentes (6). La Declaración de Bolonia de junio de 1999 llamó al establecimiento para el año 2010 de una educación superior europea que sea coherente, compatible y competitiva, de tal forma de ser atractiva para los estudiantes europeos y de otras latitudes. Originalmente se identificaron 6 líneas de acción en Bolonia, para luego agregarse tres líneas más en Praga en mayo del 2001 y una más en Berlín en septiembre del 2003 (7).

Las líneas definidas fueron:adopción de un sistema de titulaciones fácilmente comprensible y comparable, adopción de un sistema basado esencialmente en dos ciclos, establecimiento de un sistema de créditos, promoción de la movilidad, promoción de la cooperación europea en aseguramiento de la calidad, promoción de la dimensión europea en la educación superior, aprendizaje permanente, instituciones de educación superior y estudiantes, promover el atractivo del espacio europeo de educación superior, nivel de doctorado (tercer ciclo). El proceso de Bolonia fue enfocado a los gobiernos responsables de la educación superior en sus países y también a universidades, asociaciones empresariales y redes. En este modelo se determinó que los logros de aprendizaje se describen en términos de competencias, vale decir, lo que una persona sabe o es capaz de demostrar tras la finalización de un proceso de aprendizaje, las competencias constituyen puntos de referencia para el diseño y evaluación curricular, permitiendo flexibilidad y autonomía en la construcción de los planes de estudio (7).

El Proyecto Tuningse extendió hasta Latinoamérica, y entre los años 2004 y 2008 se incorporaron 18 países de la zona para esta nueva propuesta. "El proyecto Alfa Tuning 
América Latina busca "afinar" las estructuras educativas de América Latina iniciando un debate cuya meta es identificar e intercambiar información y mejorar la colaboración entre las instituciones de educación superior para el desarrollo de la calidad, efectividad y transparencia (2)".

Para la implementación del proyecto en Latinoamerica se definieron los siguientes objetivos:

- Contribuir al desarrollo de titulaciones fácilmente comparables y comprensibles en una forma articulada en toda América Latina.

- Impulsar, a escala latinoamericana, un importante nivel de convergencia de la educación superior en doce áreas temáticas (Administración de Empresas, Arquitectura, Derecho, Educación, Enfermería, Física, Geología, Historia, Ingeniería Civil, Matemáticas y Química) mediante las definiciones aceptadas en común de resultados profesionales y de aprendizaje.

- Desarrollar perfiles profesionales en términos de competencias genéricas y relativas a cada área de estudios incluyendo destrezas, conocimientos y contenido en las cuatro áreas temáticas que incluye el proyecto (2).

En el año 2010 se aprueba la segunda fase del proyecto Tuning Latinoamericano por los periodos 2011-2013 cuyo lema fue "Innovación Educativa y Social" y que busca mantener el debate iniciado con la primera fase de este proyecto.

En el 2003, el Consejo de Rectores de las Universidades de Chile (CRUCH) declaran la necesidad de modificar el sistema universitario chileno hacia un Sistema de Créditos Transferibles (SCT) (8).

En respuesta a esto, el Programa de Mejoramiento de la Calidad y la Equidad de la Educación Superior (MECESUP) realizó la convocatoria para los proyectos de renovación curricular (8).

La facultad de medicina se adjudicó el proyecto MECESUP, con el cual desarrolló el rediseño curricular de las 8 carreras pertenecientes a la facultad: enfermería, medicina, kinesiología, obstetricia, fonoaudiología, terapia ocupacional, nutrición y tecnología médica.

La facultad de medicina de la Universidad de Chile realizó adaptaciones al modelo original, dentro de las cuales se encuentra una aproximación diferente al aprendizaje por competencias, según la cual, el aprendizaje por competencias tiene por objetivo que el estudiante demuestre un saber actuar de manera pertinente a diversas situaciones y contextos, para enfrentar problemas propios de la profesión y la ciudadanía con un claro criterio de calidad, para lo cual se articulan y movilizan recursos internos, de contexto y de redes estando en condiciones de dar razón de sus decisiones y actuaciones y haciéndose cargo de los efectos e impactos de los mismos (9).

\section{Experiencia de implementación de la formación por competencias en la Escuela de Enfermería de la Universidad de Chile}

Entre los años 2006 y 2010 se elaboraron documentos en relación con la formación por competencias, entre los cuáles se contaban con el perfil de egreso, declaración de algunas competencias y sub-competencias e indicadores de logro. En el año 2011, se actualizaron los documentos mencionados.

A partir del año 2011 la Escuela de Enfermería de la Universidad de Chile, inició una reestructuración de su cuerpo académico. Como consecuencia de ello, hubo cambios en los integrantes de comisión de innovación curricular, por cual fue necesario establecer un programa de orientación y actualización de tal forma de dar los espacios para análisis, reflexión y unificación de criterios de los participantes.

Los objetivos trazados para este período 2011-2013 fueron:

- Implementar un proceso de resignificación y de participación de las académicas y estudiantes de la escuela en el proceso de innovación curricular.

- Ejecutar las actividades establecidas por la facultad para las distintas etapas del diseño del proceso de innovación curricular en la Escuela de Enfermería.

El trabajo de rediseño se organizó considerando cuatro actores relevantes:

- $\mathbb{L}$ a Comisión de Innovación Curricular como tal.

- El cuerpo académico de la Escuela de Enfermería Universidad de Chile.

- El Instituto de Ciencias Biomédicas de la Facultad de Medicina, quienes son los responsables de ofrecer los cursos de ciencias básicas.

- Llos estudiantes.

\section{Rol de cada uno de los actores del proceso}

\section{Comisión Innovación Curricular}

La responsable de esta comisión fue la subdirectora de escuela, esto se encuentra en el manual de descripción de funciones de la escuela de enfermería, las funciones de la comisión fueron:

- Coordinar las acciones de análisis, diseño e implementación de las innovaciones en cada mención en particular y en la escuela en general.

- Coordinar, difundir e informar de las diferentes instancias de habilitación docente para el proceso.

- Informar al claustro y a la dirección de la escuela sobre los diferentes aspectos del proceso de innovación.

- Colaborar en la toma de decisiones del nuevo itinerario formativo para la carrera. 
La comisión estuvo integrada por académicos de la Escuela de Enfermería Universidad de Chile y por estudiantes de los diferentes niveles de la carrera. Esta estructura aún se mantiene en funcionamiento con la finalidad de un mejoramiento continuo del proceso. En el año 2013, se integró la Coordinadora del Primer Año Innovado.

\section{Cuerpo académico de la Escuela de Enfermería, Universidad de Chile}

En el periodo 2010-2011, se realizaron nuevas contrataciones de académicos, lo que se tradujo en un $45 \%$ de nuevos docentes incorporados a la institución, los cuales contaban con diferentes grados de experiencia y provenían de diversas áreas disciplinares.

En este marco se hizo necesario realizar un levantamiento de información entre los docentes respecto a su preparación en el área de currículo por competencias, para así generar un programa de habilitación docente en este tema, el cual incluyó conceptualización, semántica, un cambio de mirada y de lenguaje entre los currículos por objetivos y por competencias, metodologías innovadoras, evaluación, que permitió a los académicos sentirse parte de un proyecto colectivo.

En la formación antes mencionada se excluyeron aquellos académicos que se encontraban cursando estudios de magíster en docencia, puesto que, en el caso de los programas de este tipo en Chile, el currículo basado en competencias es parte de los contenidos. El trabajo de la habilitación docente en la Escuela de Enfermería se programó de acuerdo a los recursos disponibles; de la propia escuela, la facultad y la vicerrectoría de la universidad. A continuación, se detallan las características de cada tipo de habilitación:

\section{- WHabilitación por vicerrectoría}

La vicerrectoría de la Universidad de Chile ofrece el programa de "Diploma Docencia Universitaria Basada en Competencias" en el cual durante los años 2010 y 2011, han sido beneficiados 15 académicos la Escuela de Enfermería, en la actualidad este diploma es obligatorio para los profesores que ingresan a la escuela y que no cuenten con una formación actualizada en la materia, esta actividad es guiada por la docente a cargo de la comisión.

\section{- GHabilitación por facultad}

La facultad posee el Departamento de Educación en Ciencias de la Salud quienes anualmente programan diferentes cursos de actualización en temas del área, los cuales son asequibles para todos los docentes. La unidad cuenta con académicos que han efectuado cursos en temas como: evaluación auténtica, introducción a la innovación, construcción de preguntas de múltiple selección, razonamiento crítico, entre otros. Este recurso se continúa utilizando por los académicos de la escuela con el fin de mantener la actualización en el tema.

- LHabilitación por la escuela

En la Escuela de Enfermería se han realizado cursos contando con recursos propios de académicos que se han formado en el área como también se ha invitado a expertos para realizar actualización en algunos temas. Durante el año 2011 se efectúo el curso "Aprendizaje basado en Problemas" al cual asistieron más del 90\% de las docentes, el 2012 el taller de "Pruebas de preguntas de selección múltiples" y también el taller "Elaboración de instrumentos de evaluación prácticas clínicas, rúbricas”.

Durante el año 2012 se mantuvo el programa de habilitación docente debido a que es indispensable que los docentes cuenten con las herramientas adecuadas antes de iniciar la implementación, siempre con el propósito de empoderar a los académicos para que asuman el proceso de innovación.

Se realizó un nuevo levantamiento de necesidades formativas por medio de una encuesta. De acuerdo con los resultados de la encuesta, se efectuaron los cursos-talleres "Curso de elaboración de fichas de curso", "Elaboración de programas" "Construcción de fichas de cursos I y II" "Estrategias innovadoras para la resolución de problemas científicos en el proceso de desarrollo profesional "Evaluación auténtica, Razonamiento clínico, Construcción de programas. Análisis de casos I y II. Construcción de mapas conceptuales. Habilidades clínicas. Diploma de docencia basado en competencia. Diploma en uso de tecnología de comunicación. Durante el presente año 2016, la habilitación se mantiene utilizando todos los recursos disponibles e incorporando a los académicos que ingresan.

\section{- Wiempo protegido}

Otro aspecto que se consideró con el claustro de igual relevancia que su habilitación, fue establecer un horario protegido de trabajo; para esto se asignó a los académicos una tarde a la semana durante las jornadas laborales, con el objetivo de analizar las diferentes propuestas que se generaban en la comisión de innovación curricular y proponer modificaciones.

\section{Instituto de Ciencias Biomédicas de la Facultad de Medicina}

El Instituto de Ciencias Biomédicas (ICBM) ofrece la formación en las ciencias básicas en la carrera de enfermería de la facultad de medicina.La comisión de innovación curricular organizó subcomisiones integradas por una docente del claustro y una docente de la comisión, con el fin de establecer un puente para el diálogo del instituto antes mencionado. El trabajo consistió en la integración de las asignaturas de acuerdo a la propuesta de malla elaborada por enfermería, fichas de curso y elaboración en conjunto de los programas. Los docentes de la escuela deben reportar periódicamente a la comisión.

Durante los meses de marzo y abril de 2011 se realizó un informe de los indicadores de logros elaborados por ICBM, que correspondieron a 12 materias de ciencias básicas del área (biológica, química y matemática). Cuyo objetivo era definir el nivel de complejidad del indicador, de acuerdo a la matriz curricular de enfermería compuesta por cinco dominios con sus competencias, sub-competencias e 
indicadores de logro generada en la comisión. Este proceso se logró completar con diversas entrevistas sostenidas con los profesores encargados de las asignaturas en un diálogo constructivo. Posteriormente a esta etapa, se inició un período de negociación con el objetivo de llegar a acuerdo sobre los créditos disponibles para las ciencias básicas en la malla del plan de formación realizada por la comisión y validada por el claustro. En la actualidad se encuentran trabajando en conjunto con la escuela en la elaboración de los programas del próximo año.

\section{Estudiantes de la Escuela de Enfermería}

La participación de los estudiantes y egresados en este proceso es importante, porque la visión que pueden aportar enriquece el proyecto. Es por esto que se le ha invitado a participar en las reuniones que realiza la comisión de innovación curricular, se han modificados los horarios para favorecer su asistencia y se han buscado diferentes estrategias para poder contar con su presencia, a veces por la carga académica no ha sido tan constante como quisiéramos, de igual forma son invitados con tiempos protegidos a las jornadas que se programan con la finalidad de analizar nuevas propuestas y de esta forma ellos puedan tener voz en el proceso, como también para validar los diferentes hitos que implica. Otra medida utilizada para abrir este espacio a los estudiantes ha sido efectuar reuniones a cargo de la responsable de la comisión con los representantes de las diferentes estructuras que poseen, con la finalidad de mantenerlos al día. Los estudiantes han estado presentes en la validación del perfil de egreso, participaron en la validación de la malla, han aportado en relación a contenidos de asignaturas proponiendo nuevas temáticas para ser consideradas desde la relevancia que observan en sus experiencia clínica. Sin duda la participación de los estudiantes es relevante y necesaria en el proceso.

\section{Fases del proceso de construcción curricular}

Dentro de la fase de diseño curricular utilizado en la facultad de medicina de la Universidad de Chile (9) se cuenta con varias fases:

1. Levantamiento de la necesidad formativa

2. Diseño del perfil de egreso

3. Validación del perfil de egreso

4. Organización y estructura de la matriz de competencias

5. Construcción de la estructura formal del currículo

En relación con estas se presentan las siguientes fases:

Fase 1: "Levantamiento de la necesidad formativa" En esta fase se recogió información desde diferentes actores, vale decir, gremios empleadores, lineamientos nacionales e internacionales, docentes, egresados, estudiantes.

Fase 2: "Diseño del perfil de egreso".

Con la información recogida, la comisión diseñó un perfil de egreso, considerando para esto como engranaje fundamental la filosofía de la disciplina y de la institución. Para estos efectos comprendemos que el perfil de egreso como la "Promesa que hacemos a la sociedad".

Se resignificó y redefinió el perfil de egresado de enfermería, para esto se realizaron las siguientes actividades:

- Consulta individual

- Presentación en el claustro en diferentes reuniones generales y tiempo protegido

- Reformulación del perfil con los insumos aportados por las diferentes instancias

Fase 3: "Validación del perfil de egreso"

Esto se realizó a través de:

- Jornada de validación del perfil de egreso en conjunto con estudiantes junio 2011

- Revalidación por expertos

En la declaración del perfil de egreso van incluidos aquellos dominios, que son todas las acciones que se expresan en competencias y en las cuales las instituciones se comprometen a habilitar al estudiante de la carrera de enfermería. También el dominio va declarado en términos de las competencias y sub-competencias, material que se actualizó y que han sufrido modificaciones de acuerdo con los nuevos requerimientos.

En el año 2011 se realiza una revisión de los dominios anteriormente planteados, validándose posteriormente con el claustro, quedando conformados por:

- Dominio específico o troncal: Gestión del cuidado que se sustenta en un marco jurídico, marco político y social, en el desarrollo de la disciplina y en el marco de la institucional de la Universidad de Chile.

A este dominio troncal lo alimentan cuatro dominios:

1. Dominio gestión en salud

2. Dominio en educación

3. Dominio de investigación

4. Dominio genérico transversal; donde se encuentra el desarrollo personal, la ética y bioética.

Fase 4: "Organización y estructura de la matriz de competencias"

Una vez validado el perfil de egreso, se continuó con el trabajo que se venía desarrollando desde la comisión de innovación curricular en relación a la elaboración de la hipótesis de progresión del aprendizaje junto a la revisión de la matriz de contenidos, declaradas con las competencias, sub-competencias, indicadores de logro. La comisión acuerda que la hipótesis de progresión se realizará de acuerdo con el ciclo vital y nivel de complejidad.

La organización de los dominios se debe considerar en relación al tiempo que se dispone en el plan de formación que corresponde a 300 créditos, un crédito equivale a 27 horas, de acuerdo con el peso relativo que se le ha asignado a cada uno. 
Esta organización permite ir confeccionando y actualizando la matriz, la cual debe considerar el nivel de complejidad de cada competencia y sub-competencia; junto con eso se elaboran los indicadores de logro para cada una de ellas y su vinculación con los niveles de formación.

Fase 5: "Construcción de la estructura formal del currículo"

La expresión gráfica de la fase del diseño es la elaboración de la malla curricular; a la propuesta existente; en el año 2012 se le realiza una restructuración luego de negociaciones con el Instituto de Ciencias Biomédicas donde se realiza la denominación de las asignaturas y se les otorga los créditos de acuerdo con las competencias que se abordan y el nivel de complejidad. La facultad da directrices generales a tener en cuenta, cada año debe tener un total de 60 créditos, el idioma inglés pasa a ser obligatorio con 9 créditos a lo largo de la carrera, dos módulos integradores en quinto $5^{\circ}$ y $8^{\circ}$ semestre y 4 cursos de formación general de 2 créditos c/u.

En Julio del 2012 se realizó una jornada extramural, para el cuerpo académico y estudiantes seleccionados; en esa oportunidad el objetivo fue socializar la malla y realizar un análisis en conjunto. Así se fue estructurando y realizando modificaciones durante todo ese año para posteriormente ser validada en diferentes espacios; dirección de escuela, cuerpo académico, con los estudiantes, a los directivos del ICBM y el consejo de directivo de la facultad.

En noviembre 2012: se cumplió un nuevo hito la "Validación de Malla Curricular" proceso que se lleva a cabo en consejo de facultad, entidad de alta jerarquía con poder resolutivo.

La nueva malla (Tabla 1) espera que los estudiantes se formen desde una construcción curricular integradora, la cual supone un proceso de planificación holística y helicoidal en el cual convergen elementos cualitativos y cuantitativos, de manera armónica como partes inseparables e interactuantes del todo que es la formación de profesionales que conocen las especificidades de la integración disciplinar, interdisciplinar y transdisciplinar $(10,11)$. Esto se traduce en un rediseño que consideró el ciclo vital, niveles de atención, trabajo intersectorial, red asistencial; y permitirá desarrollar el juicio profesional,

Tabla 1. Currículo Basado en Competencias. Escuela de Enfermería Universidad de Chile.

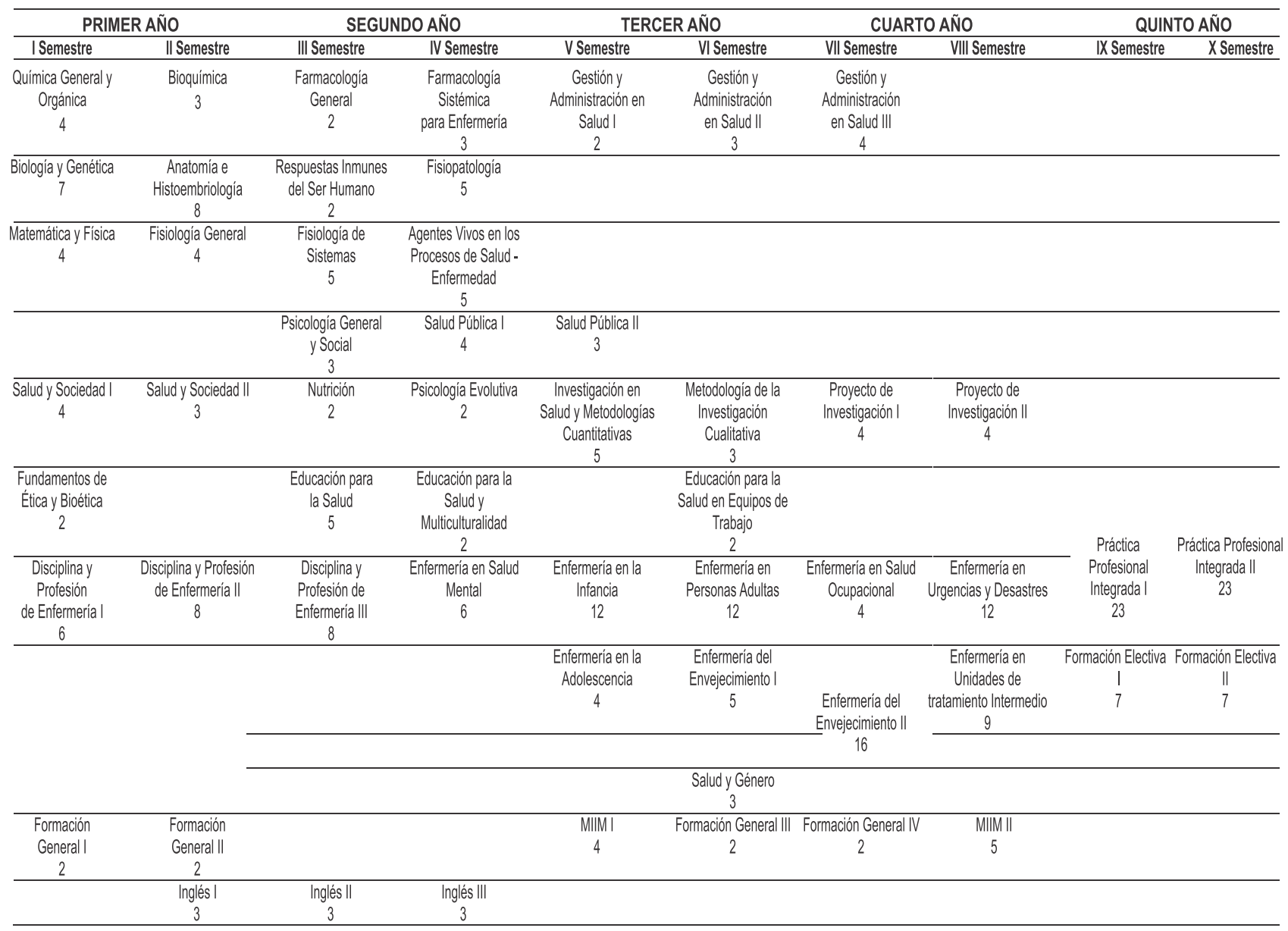

Fuente: Elaboración propia del autor. 
aplicando el proceso de atención de enfermería en forma progresiva. Por ello, la escuela adelantará la práctica profesional con personas sanas en el segundo semestre del primer año, profundizará áreas existentes e incorporará otras nuevas. Entre ellas: Enfermería en la adolescencia, grupo etario que tiene demandas propias de salud, que requiere un mayor trabajo de promoción y prevención con una mirada de largo plazo; Enfermería en el Envejecimiento, considera el perfil epidemiológico del país, con un número creciente de adultos mayores, por lo que esta área demanda mayor profundización; Salud y Género, contribuirá a formar a los estudiantes en políticas de salud y género, a fin que puedan aportar desde la enfermería en el tema; Salud Ocupacional, por la preparación, conocimientos y cualidades organizativas, se orientará a los nuevos profesionales a actuar como eje integrador en la promoción de salud de los trabajadores y en la prevención de riesgos laborales; y Educación y Multiculturalidad, dado que Chile cuenta con diferentes etnias y con medio millar de inmigrantes, abordará el tema desde un ámbito integrador para dar respuesta a las demandas de salud de estos grupos.

La transversalidad de Salud Mental y de Ética que cuentan con asignaturas propias, pero a la vez integran todo el canal profesional.

Efectuado el proceso de validación de la malla, el trabajo apuntó a la elaboración de las fichas de curso para lo cual fueron designados los profesores que se harán cargo de las asignaturas del primer año innovado. Posteriormente se inicia la confección de los programas de asignatura de acuerdo con la matriz y la hipótesis de progresión realizadas efectuando la determinación de las unidades de aprendizaje y asignando el tiempo presencial y no presencial que se debe considerar para evidenciar un resultado de aprendizaje deseado .La comisión cumple un rol de orientador del proceso a los profesores encargados de las asignaturas, respetando su autonomía y brindando apoyo si es necesario para mantener los lineamientos planteados en el trabajo en conjunto.

\section{Otras actividades de la comisión}

Elaboración y revisión del reglamento

Se revisaron y actualizaron los planes de formación conducentes a la licenciatura en enfermería y al título profesional de enfermero(a) otorgado por la facultad de medicina, Universidad de Chile.

\section{Elaboración del Plan de Formación de Enfermería}

Es el documento oficial de la carrera y contiene: propósito formativo de la carrera de enfermería, perfil de egreso, dominios y competencias, la malla curricular, cuadro de actividades curriculares, que contempla periodo, dominio/formación especializada, asignatura, línea formativa, flexibilidad curricular, tipo de curso, requisitos y créditos. El plan de formación fue validado en las diferentes instancias.
Propuesta de recursos docentes

A solicitud de dirección y subdirección de la escuela se realiza una propuesta de la necesidad de recursos docentes a través de una proyección del cálculo de horas teóricas, de talleres o seminarios y de acuerdo con las demandas necesarias para cubrir las metodologías innovadoras que se aplican en un currículo basado en competencias.

\section{Definición del horario}

Para cumplir con esta solicitud se realiza un trabajo en conjunto con todos los profesores encargados de primer año innovado, se diseña un horario manteniendo el criterio un $50 \%$ de la jornada presencial y un $50 \%$ no presencial.

\section{Trabajo actual de innovación curricular}

La escuela de enfermería de la Universidad de Chile en marzo de 2013 ha iniciado la implementación del nuevo currículo de formación basado en competencias; durante su transcurso se ha ido realizando en forma paralela una evaluación del desempeño en los diferentes actores, se ha realizado una evaluación desde facultad y dentro de la comisión se está visualizando el inicio del proceso de evaluación formal del currículo.

Frente a una consulta realizada a una estudiante de enfermería que voluntariamente optó por iniciar nuevamente su formación en el programa innovado ha declarado: "existe una diferencia clara en la carga horaria, además de que también en primer año ya no existe una formación basada sólo en las ciencias, sino que ahora es más integral entre lo que son los ramos humanistas con la base científica. Esto es un gran avance, porque te plantea desde un comienzo lo que es el deber y el hacer de la enfermera(o). Asimismo, las metodologías de enseñanza son más lúdicas, tenemos discusiones en grupo y existen más trabajos de investigación. Ahora nos sentimos agentes responsables de nuestra carrera desde el primer año" $(10,11)$.

\section{Conclusiones}

El currículo basado en competencias pone en el centro del proceso al estudiante, su rol es activo, por lo tanto, el profesor-tutor deberá potenciar no sólo lo cognitivo sino también todas aquellas habilidades que habrá de poner en marcha frente a situaciones que necesitará resolver y que se les presentarán tanto en su formación como cuando desarrolló su rol profesional. El papel del docente es fundamental, por lo cual debe estar preparado.

El proceso de cambio curricular lleva implícita una serie de actividades, que tienen un orden secuencial, pese a ellos también se deben efectuar otras acciones en forma paralela como por ejemplo el fortalecimiento de los equipos de trabajo, un pilar fundamental es el cuerpo académico de las instituciones en quienes se debe poner el énfasis a través de un programa de habilitación docente que se base en las necesidades reales de formación, como resultó ser una gran fortaleza en la experiencia vivida en la Escuela de 
Enfermería Universidad de Chile, generando un cambio de lenguaje y de mirada.

Los cambios en este nuevo plan de formación de la Escuela de Enfermería de la Universidad de Chile, permiten una mirada más integradora del aprendizaje, considerando ciclo vital, niveles de atención, trabajo intersectorial, red asistencial; y permitirá desarrollar el juicio profesional, aplicando el proceso de atención de enfermería en forma progresiva., considerando para esto, como se mencionó, el rediseño curricular integrador.

Una de las claves del éxito de un cambio de currículo debe considerar el trabajo desde las bases, realizar un trabajo cooperativo, socializado, discutido y llegando a consenso con todo el cuerpo académico que conforma esa unidad. Además, es relevante hacer mención a la importancia de validar todos los hitos por diferentes actores internos y externos a la institución. En este sentido la Escuela de Enfermería cuenta con un comité consultivo conformado por profesionales enfermeras de diferentes centros asistenciales que analizan y dan sugerencia a los procesos que lleva a cabo la unidad. En la experiencia vivida en la Escuela de Enfermería de la Universidad de Chile, se ha intentado resguardar la participación del cuerpo académico en todos los procesos, salvo en algunas ocasiones que por cumplimiento de plazos ha tenido que resolver la comisión y usar las diferentes vías de información para socializar.

Otro eje importante en el proceso es considerar la participación de los estudiantes; su aporte permite contar con una opinión desde la vivencia de la implementación.

\section{Conflicto de intereses}

Los autores manifiestan que no tienen ningún conflicto de interés.

\section{Referencias}

1. Irigoyen-Morales JJ, Jiménez MY, Acuña KF. Competencias y educación superior. RMIE2011; 16(48), 243-266. Disponible en: <http://www.scielo.org.mx/ scielo.php?script=sci_arttext\&pid=S140566662011000100011\&lng=es\&nrm=iso>.

2. Dannar P. Millennials: what they offer our organizations and how leaders can maeke sure they deliver. JVBL 2013; 6(1):1-12. Disponible en: http://scholar. valpo.edu/cgi/ viewcontent.cgi? article $=1073$ \&context=jvbl

3. Alfa EuropeAid Co-Operation Office. Tunnig América Látina, Innovación educativa y social 2011-2013. Disponible en: http://www.tuningal.org/es

4. Posada R. "Formación superior basada en competencias, interdisciplinariedad y trabajo autónomo del estudiante", Revista Iberoamericana de Educación 2004. Disponible en: https://www.ugr.es/ ugr_unt/ Material\% 20M\%F3dulo\%205/competencias_univ.pdf
5. Tobón S. La formación basada en competencias en la educación superior: el enfoque complejo. Universidad Autónoma de Guadalajara. 2008. Disponible en: http://cmapspublic3.ihmc.us/rid=1LVT09TXFX1VKC0TM-16YT/Formaci\%C3\%B3n\%20basada\% 20en\%20competencias\%20(Sergio\%20Tob\%C3\%B3n). pdf

6. González J, Wagenaar R. Universities' contribution to the Bologna Process, An introduction. The Tuning Project. 2nd edition. España, Publicaciones de la Universidad de Deusto, febrero de 2008. Disponible en: http://www.unideusto.org/tuningeu/images/stories/Public ations/ENGLISH BROCHURE FOR WEBSITE.pdf

7. Gonzalez J, Wagenaar R. Tuning Educational Structure in Europe. The Tuning Project. España, Publicaciones de la Universidad de Deusto, diciembre de 2003. Disponible en: http://www.uc.pt/ge3s/event_04/event_04/ tuningexecutivesummary-3.pdf

8. Tumanoff R., Chauriye-Batarce S. Innovación Curricular en las Universidades del Consejo de Rectores 20002010.Santiago de Chile, Consejo de Rectores de las Universidades Chilenas (CRUCH), noviembre de 2011. Disponible en: http://sct-chile.consejoderectores.cl/ documentos_WEB/Innovacion_Curricular/2.Informe_IN NOVACION_CURRICULAR.pdf

9. Facultad de medicina, Universidad de Chile [Internet]. Santiago de Chile: Innovación curricular [citado jul 2013]. Nuestros desafíos [aprox. 2 pantallas]. Disponible en: http://www.med.uchile.cl/innovacion/innovacion/nuestros -desafios.html

10. Bustos R. La innovación curricular dentro de las escuelas. El pulso. 7 de mayo de 2013 Disponible en: http://elpulso.med.uchile.cl/20130507/noticia9.html

11. Serrudo-Ormachea M. La Construcción Curricular basada en el Pensamiento Complejo. Revista de Psicología 2012; 7:31-41 Disponible en: $<$ http://www.revistasbolivianas.org.bo/scielo.php?script= sci_arttext\&pid=S2223-30322012000100003\&Ing=es\& $\mathrm{nrm}=$ iso $>$. 